\title{
Why the incidence in dialysis in the Lisbon and Tagus Valley Region (LVTR) is two to three times higher than in other regions of the country
}

\author{
M. Martins Prata \\ Professor of Nephrology
}

\section{ABSTRACT}

The analysis of data from the Ministry of Health's Integrated Chronic Kidney Disease Management Platform (PI-GID), reveals the existence of a striking regional difference in the incidence in dialysis. This difference is characterized by high levels of incidence in all regions, except the Alentejo, and extremely high levels in the Lisbon and Tagus Valley region. In this region, the incidence values are two to three times higher than in the others. The difference between regions is the result of a different number of patients starting hemodialysis, since the incidence in peritoneal dialysis is low and similar in all of them. It is difficult to find justification for such a discrepancy since the prevalence of factors usually responsible for the higher or lower level of incidence in dialysis does not differ significantly between them. A probable explanation may lie in the difference found in the concentration of public hemodialysis centers, a fact that may determine a difference in the criteria used for the entry of patients undergoing substitutive treatment of renal function. In fact, we found a close correlation between the number of public hemodialysis centers per million inhabitants and the level of incidence in dialysis. A more comprehensive analysis of the data from the Ministry of Health's Integrated Chronic Kidney Disease Management Platform (PI-GID), if allowed, would be of enormous interest, not only to explain this discrepancy in the incidence of dialysis between regions, but because it could probably allow an explanation for Portugal having (one of) the highest levels of dialysis incidence in Europe

Key-words: Chronic Kidney Disease, dialysis, incidence, prevalence

C 2021 Portuguese Journal of Nephrology \& Hypertension. Published by Publicações Ciência \& Vida This is an open access article under the CC BY-NC-ND license (http://creativecommons.org/licenses/by-nc-nd/4.0/).

\section{INTRODUCTION AND RESULTS}

The incidence of dialysis in Portugal is much higher than that registered in most other countries in Europe, and the cause or causes are not completely understood ${ }^{1}$.

During the last few years, a higher prevalence of diseases that cause chronic kidney disease and their progression, mainly diabetes mellitus and arterial hypertension, has been identified as the responsible cause. However, the existing data does not confirm this. Spain, for example, with a very similar prevalence of these pathologies 2,3 has approximately half the number of patients per million inhabitants of the population ( $\mathrm{pmp}$ ) starting dialysis per year: on average, $130 \mathrm{pmp}$ compared to the $244 \mathrm{pmp}$ observed in Portugal.

Today, it is known that many other factors may be involved - medical and non-medical factors - and that countries with similar prevalences of those pathologies may present significant differences between themselves in the number of patients starting renal replacement therapy.
For this reason, international health entities ${ }^{4}$ have insisted that in any country or region where there is a significant difference in the levels of the prevalence of chronic kidney disease, or of the incidence in dialysis, it is essential to find the cause, or causes, responsible, in order to be able to implement the appropriate corrective measures.

A recently published paper ${ }^{5}$, the first to be carried out based on data from the Ministry of Health's Integrated Chronic Kidney Disease Management Platform (PI-GID), reveals that the difference in incidence in dialysis does not exist only in relation to other countries. The authors demonstrated, for the first time, the existence of a striking regional difference in the incidence of dialysis in Portugal.

As is easily understood, it is so much more important to know the cause or causes that justify the differences in incidence in dialysis between the five regions of the country, as it is to know why Portugal has an incidence far above the European and world average.

1. Incidence in dialysis in the Lisbon and Tagus Valley Region (LVTR) and in the other four regions 
According to de Almeida E. et $\mathrm{al}^{5}$, LVTR had a dialysis incidence of $386.7 \mathrm{pmp}$ in 2016, 1.8 to 3.1 times higher than that of the other regions. These high values persist throughout the period of analysis, from 2010 to 2016 (Figure1).
However, this difference between regions is observed only in the hemodialysis sector, since the incidence in peritoneal dialysis is very similar in all regions (Figure 2).

\section{Figure 1}

Regional Incidence (pmp) in dialysis (2010 - 2016)

pmp

400

379

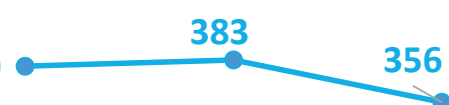
356 386 361 387 300

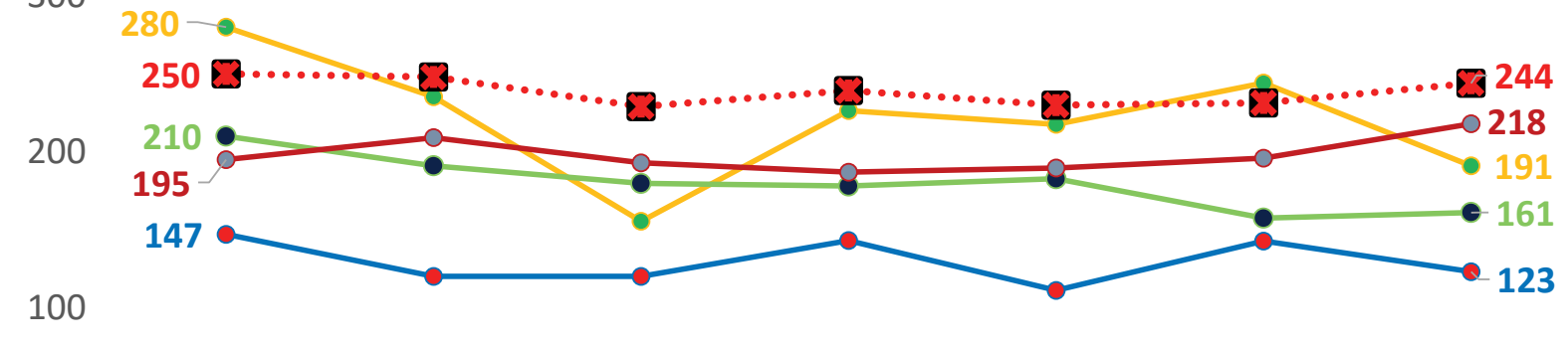

0

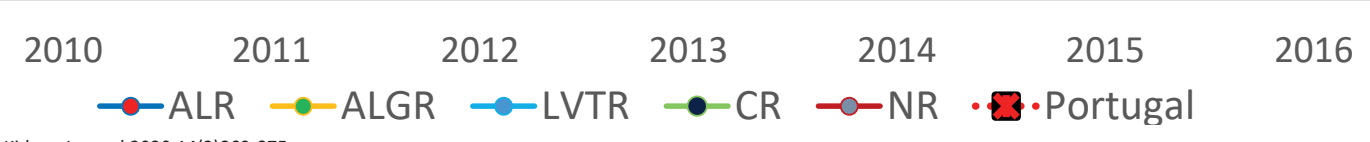

From: de Almeida E et al: Clinical Kidney Journal 2020;14(3)869-875

\section{Figure 2}

Regional Incidence in Hemodialysis (a) and Peritoneal Dialysis (b) (pmp)

( a )

$\mathrm{pmp}$

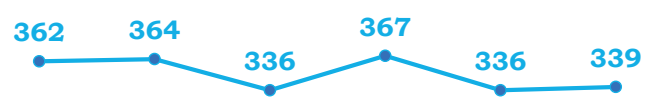

300

200

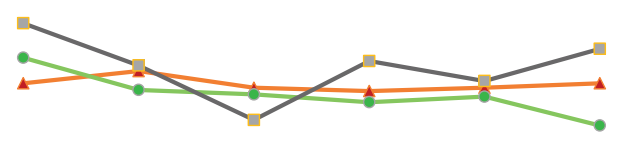

100

0

$$
\begin{array}{llllll}
2010 & 2011 & 2012 & 2013 & 2014 & 2015
\end{array}
$$

$\rightarrow-$ LVTR $\rightarrow$-North R $\rightarrow$-Center R ---South

From: SPN Dialysis Registry, 2016

(b)

pmp

40

30

20

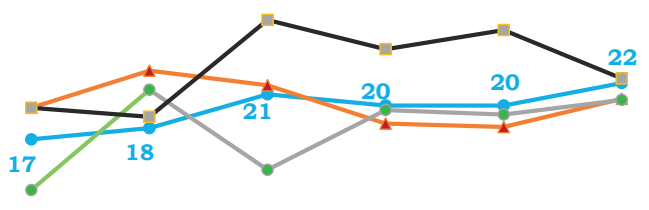

10

0
2010
2011
2012
$2013 \quad 2014$
2015
$\leadsto$ LVTR $₫$ North $\rightarrow$ Center R --South 
In summary, the LVTR has a high incidence of dialysis, much higher than that observed in other regions of the country, and this difference is due exclusively to the much higher number of patients who undergo hemodialysis.

\section{CAUSE, OR CAUSES, THAT MAY JUSTIFY THE HIGHER INCIDENCE IN HEMODIALYSIS IN THE LISBON AND TAGUS VALLEY REGION}

Among the factors that may influence the incidence of dialysis, the most frequently mentioned are:

a) age of the population.

b) risk factors (RF) for chronic kidney disease (CKD) and its progression: diabetes mellitus, high blood pressure, obesity, salt intake, smoking and physical inactivity. c) prevalence of chronic kidney disease in the population

d) early initiation of dialysis.

e) accessibility, availability and characteristics of the health service in the renal area.

a) The percentage of the population in which chronic kidney disease is more frequent - population aged 65 and over - is very similar throughout the regions. It varies from $19.5 \%$ in the North Region to $25 \%$ in Alentejo. In the LVTR it is $21.3 \%$. (Figure $3(\mathrm{a})$ ) Between 2009 and 2018 the average age of the incident patient population was always lower in the LVTR. (Figure 3(b). -

b) The prevalence of risk factors (RF) for chronic kidney disease (CKD) and their progression are very similar in all regions (Table I).

The LTVR population has a slightly higher prevalence of diabetes. However, according to data from the Portuguese Society of Nephrology registry, this region has a lower incidence and prevalence

\section{Figure 3}

a) Population: age $>=65$ years old

$\%$

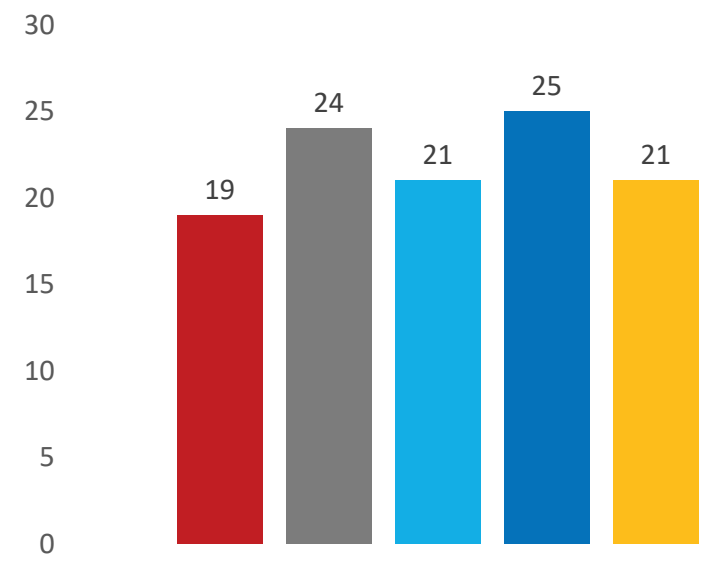

घ North $\mathrm{R} \backsim$ Center $\mathrm{R} \backsim$ LVTR $\square$ Alentejo $\mathrm{R} \backsim$ Algarve $\mathrm{R}$

From: Health Profiles of Portugal Administrative Regions b) Average age of incident patients in dialysis

years 70

65

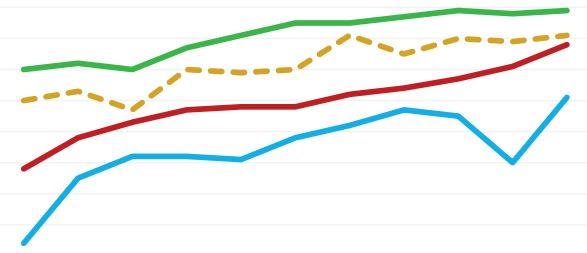

60

\begin{tabular}{c|c|c|c|c|c|c|c|c|c|c|c|}
55 & 20 & 20 & 20 & 20 & 20 & 20 & 20 & 20 & 20 & 20 & 20 \\
& 09 & 10 & 11 & 12 & 13 & 14 & 15 & 16 & 17 & 18 & 19 \\
\hline -NR & 65 & 66 & 66 & 67 & 67 & 67 & 67 & 67 & 68 & 68 & 69 \\
\hline - CR & 68 & 68 & 68 & 69 & 69 & 70 & 70 & 70 & 70 & 70 & 70 \\
\hline - Lisbon & 62 & 65 & 65 & 65 & 65 & 66 & 66 & 67 & 67 & 65 & 67 \\
\hline - - South & 67 & 67 & 67 & 68 & 68 & 68 & 69 & 69 & 69 & 69 & 69
\end{tabular}

From: PSN - Dialysis and Transplantation Registry

\section{Table I}

Age $\geq 65$ years and risk factors for chronic kidney disease

\begin{tabular}{l|c|c|c|c||c||c|} 
& $\begin{array}{c}\text { Age } \\
\mathbf{2 6 5} \text { years } \\
(\%)\end{array}$ & $\begin{array}{c}\text { Diabetes } \\
\text { Prev. } \\
(\%)\end{array}$ & $\begin{array}{c}\text { Hypertension } \\
\text { Prev. } \\
(\%)\end{array}$ & $\begin{array}{c}\text { Overweight } \\
\text { Prev. } \\
(\%)\end{array}$ & $\begin{array}{c}\text { Obesity } \\
\text { Prev. } \\
(\%)\end{array}$ & $\begin{array}{c}\text { Smoking } \\
\text { Prev. } \\
(\%)\end{array}$ \\
\hline North R & 19.5 & 9.8 & 32.9 & 35.6 & 15.3 & 17.1 \\
Center R & 23.6 & 8.3 & 38.6 & 38.4 & 16.3 & 17.2 \\
LVT R & 21.3 & 10.5 & 32.8 & 35.7 & 15.2 & \\
Alentejo R & 25 & 10.7 & 40 & 36.1 & 15.5 & 16.8 \\
Algarve R & 21.1 & 7.7 & 32.3 & 34.3 & 12 & 17.4 \\
\end{tabular}

From: INSEF and Health Regional Profiles 
Table II

CKD(1-5) Prevalence and Incidence in dialysis (2016)

\begin{tabular}{l|c|c|c|}
\multicolumn{1}{c|}{} & Portugal (mainland) & LVT Region & Center Region \\
\hline CRD(1-5) prevalence \% & 20.72 & 18.4 & 24.9 \\
Incidence in dialysis (pmp) & 243.9 & 386.7 & 160.8
\end{tabular}

From de Almeida E et al: Clin Kidney J 2020: 14(3):869-875 and PSN Dialysis Registry

of diabetic patients in hemodialysis than the other regions. The reverse is true with regard to arterial hypertension ${ }^{6}$.

In summary, there are no significant differences in the levels of RF prevalence among the five regions that can justify the marked difference in incidence in dialysis in LVTR.

c) Regarding the prevalence of chronic kidney disease in the Portuguese population, according to the only published study in which the prevalence of CKD stages 1-5 are presented by region, LVTR has one of the lowest prevalences (Table II). With 18.4\%, LVTR has a much higher incidence in dialysis (386.7 pmp) than other regions that have higher prevalence of CKD stages 1-5 in the population.

d) Early initiation in dialysis: despite the importance that this factor may represent in the levels of incidence in dialysis ${ }^{7,8}$, it was not possible to determine whether it had an influence, or not, and to what degree, in the levels of incidence in dialysis in Portugal and in each of the regions. It was, therefore, impossible to verify whether the degree of renal function - one of the important reasons for the clinical decision to enter dialysis (but not the only one) - may or may not have influenced the levels of incidence in dialysis in our country.

It is important to remember that all the data needed to realize the importance of this factor are registered in the Ministry of Health's Integrated Chronic Kidney Disease Management Platform (PI-GID). However, the responsible entities - National Health Board and the National Comission for Monitoring of Dialysis - do not seem interested in carrying out or allowing such an analysis.

e) The accessibility, availability and characteristics of the National Health Service in the renal area are usually considered as factors that can significantly influence the levels of incidence in dialysis. In general, the incidence in dialysis tends to be higher in the countries, or regions, where there is a greater number of dialysis centers. This increased availability may change the clinical criteria commonly used for the entry of patients into dialysis and often induces a greater willingness to accept more elderly patients and patients who are more fragile and sick with a higher number of comorbidities ${ }^{9}$.

Portugal has an important and well-distributed network of hemodialysis centers (Table III). Public hemodialysis centers and peritoneal dialysis centers are integrated in the Public Nephrology Services which are responsible for all nephrological assistance in the hospital area. Private hemodialysis centers belong, for the most part $-87 \%$ - to large international dialysis companies (43\% Nephrocare, $30 \%$ Diaverum, $10 \%$ DaVita, $4 \%$ B. Braun and $13 \%$ others $)^{10}$.

With $12.6 \mathrm{pmp}$ and 13.4 per $10.000 \mathrm{Km} 2$, Portugal has a higher number of hemodialysis centers than that found in most other

\section{Table III}

Total, public \& private hemodialysis centers

\begin{tabular}{l|c|c|c||c} 
& Total & Public & Private & \% Priv/Publ \\
\hline North R. & 47 & 9 & 38 & 81 \\
Center R. & 18 & 4 & 14 & 78 \\
\hline LVT R. & 47 & 10 & 37 & 79 \\
\hline Alentejo R. & 8 & 1 & 7 & 87 \\
Algarve R. & 4 & 1 & 3 & 75 \\
\hline Portugal (mainland) & 124 & 25 & 99 & 80
\end{tabular}

From: Competition Authority's National Report 2020

countries in Europe. Only two countries - France and Greece - have more hemodialysis centers pmp and Portugal is the $5^{\text {th }}$ European country with more hemodialysis centers per $10,000 \mathrm{Km}^{1}$.

In Portugal, the largest number of hemodialysis centers are located in two regions, the LVTR and the North Region.

However, LVTR has a much higher number of hemodialysis centers pmp and per $10.000 \mathrm{Km} 2$ than any of the other regions, both in the public and private sectors (Figure 4).

The Alentejo, the least populated region (5\%), with one of the largest areas (30\%), has the lowest number of hemodialysis centers in the public sector: $1.4 \mathrm{pmp}$ and 0.36 per $10,000 \mathrm{~km} 2$.

In all regions, there is a clear predominance of the private sector, varying from $75 \%$ in the Algarve to $87 \%$ in the Alentejo. In the LVTR, $79 \%$ centers belong to the private sector.

The number of incident patients in hemodialysis (pmp) has a strong positive correlation with the number of public hemodialysis centers (pmp). On the other hand, the relationship with the number of private centers (pmp) is much weaker (Figure 5).

This fact is easily understandable since it is only the public centers (Nephrology Centers of Public Hospitals) that are authorized to send patients for dialysis. Hemodialysis centers in the private sector have no influence, at least in a direct way, on the number of patients who are referred for hemodialysis, since they are limited to receiving patients that public centers transfer to them.

It is evident that it would be important to know the exact number of patients advised to go on dialysis at each of the public centers. However, with the available data, it is only possible to know the average number of patients sent for dialysis at each public dialysis center, 


\section{Figure 4}

Hemodialysis Centers: pmp \& /10.000 Km2

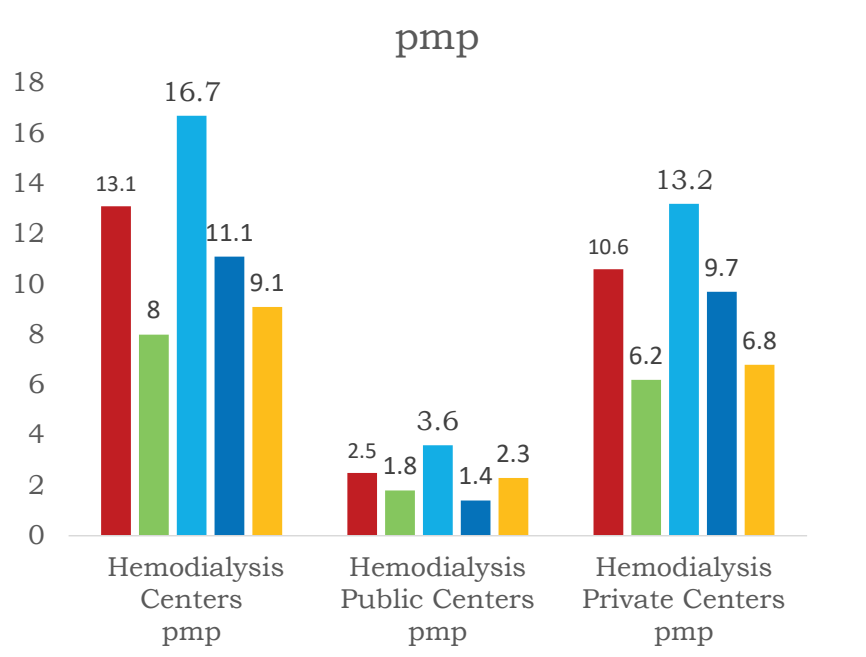

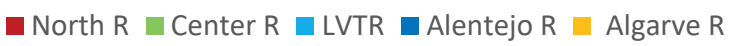

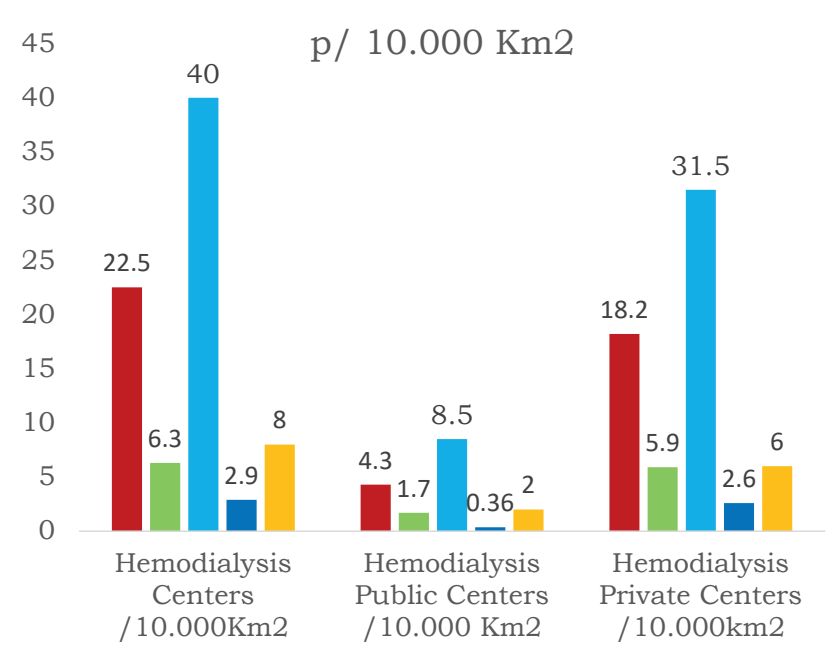

$\square$ R. Norte $\square$ R. Centro $\square$ RLVT $\square$ R. Alentejo $\square$ R. Algarve

ratio number of centers / pmp was calculated based on the population of the 2011 National Census as referred to in reference 5 From: Competition Authority's Report 2019Registry

\section{Figure 5}

a) Hemodialysis Public Centers (pmp) and Incidence in dialysis (pmp)
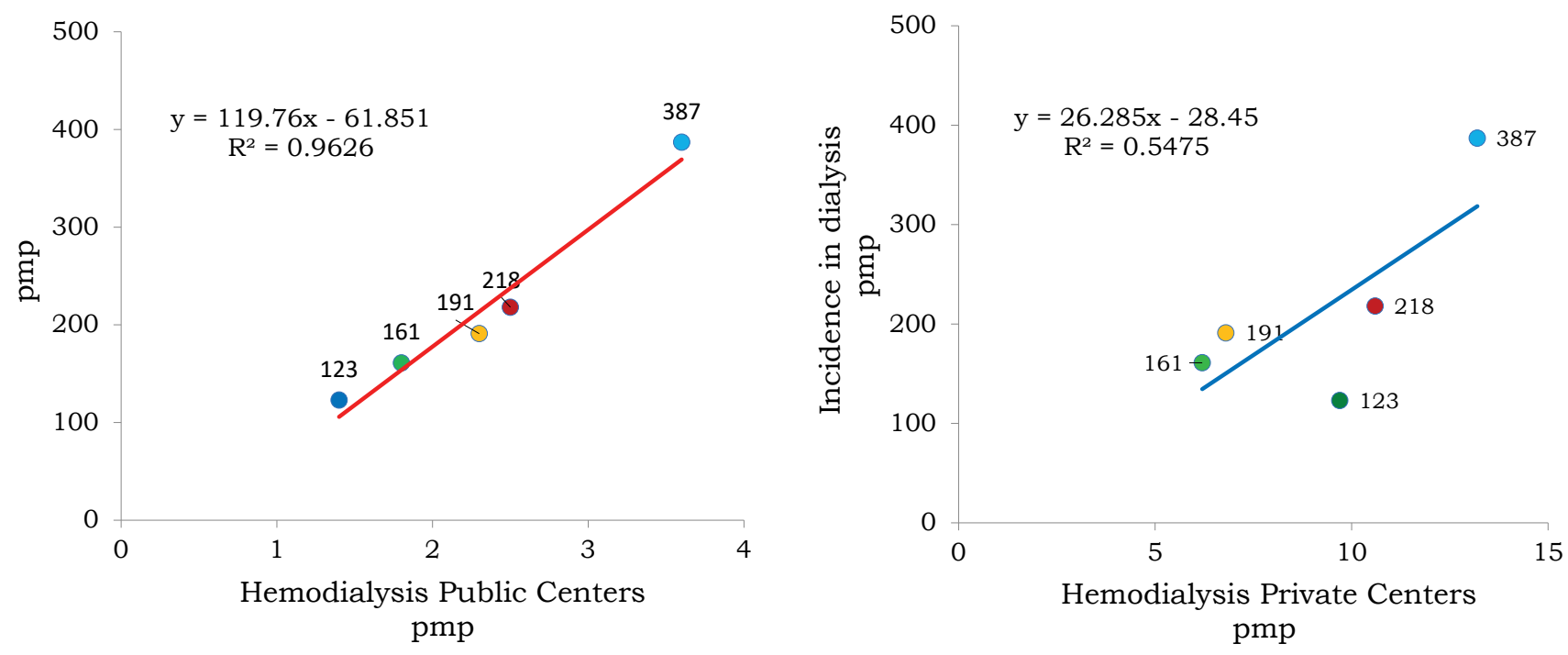

From: Competition Authority's Report 2019Registry

which varied, in 2016, from 75 patients in the NR and 102 in the CR (LVTR, 100; AIR, 80 and 96 in AIGR). However, it is very likely that the number of patients sent by each center may differ significantly from center to center, since it is known that there are frequently significantly different criteria in the medical decision to enter dialysis from team to team or even from doctor to doctor.
Without those data, it is only possible to conclude that the greater the number of public dialysis centers per million inhabitants, the higher incidence in hemodialysis. The number of private centers does not seem to influence, at least in a direct way, the value of the incidence in dialysis. However, some influence may exist as a large part of the staff - doctors and nurses - work in both public and private centers. 


\section{DISCUSSION}

Portugal (mainland) is a small country with about 10 million inhabitants and around 90 thousand square kilometers divided into five administrative regions: North (NR), Center (CR), Lisbon and the Tagus Valley (LTVR), Alentejo (ALR) and Algarve (ALGR).

It is well known that Portugal has a very high incidence in dialysis, one of the highest in Europe and in the world. In 2016, an average of 132 pmp in European countries ${ }^{11}$ and 244 pmp in Portugal.

Surprisingly, an analysis of the registration data of the Ministry of Health's Integrated Chronic Kidney Disease Management Platform (PI-GID), where data of all new incident patients must be registered by the Nephrology Centers of Public Hospitals before being admitted to dialysis, revealed a striking regional difference in incidence in dialysis 5 .

The Lisbon and the Tagus Valley Region has a dialysis incidence two to three times higher than the other regions: $386.9 \mathrm{pmp}$ compared to $122.9 \mathrm{pmp}$ in the ALR, $160.8 \mathrm{pmp}$ in $\mathrm{CR}, 190.9 \mathrm{pmp}$ in the ALGR and $218.4 \mathrm{pmp}$ in the NR.

All regions, except the ALR, have higher levels of incidence than the European average and that is only due to the incidence in one of the techniques, hemodialysis. Likewise, the difference in the incidence of dialysis in LVTR compared to the others is due only to the greater number of patients undergoing hemodialysis, since the incidence in peritoneal dialysis is similar across all regions.

In all the regions, there is a clear predominance of the private dialysis sector and it is known that the predominance of the private sector, in relation to the public, can have a very significant influence on access and the choice of type of treatment. Hörl WH et al ${ }^{12}$ carried out an international 1999 study involving data from several countries with different structures regarding the renal area of the health service, and clearly demonstrated that "provider structure influences access to and choice of ESRD treatment"; in countries with a predominance of the private sector the incidence was higher and, as regards the type of treatment performed, the incidence in hemodialysis was much higher than that of peritoneal dialysis. This is something very similar to what is observed in Portugal where $93 \%$ of patients are treated in the private sector.

However, this fact, if it can help to explain the high levels of incidence between Portugal and other European countries, can hardly explain the striking difference among the different regions of the country where, in all of them, the private sector is largely predominant.

In fact, if the difference in incidence in dialysis between Portugal and the countries of Europe is already difficult to understand, and the cause or causes are not yet completely understood, it is very likely that the same will occur when we try to understand what happens internally among regions.

It is known that the prevalence of risk factors for CKD and its progression (RF) are high in Portugal and that they may explain, at least in part, the high levels of incidence in dialysis in the country. However, although there are small differences among regions in the RF prevalence, these cannot justify such profound differences in incidence.

Likewise, there are no striking differences in the prevalence of CKD stages 1-5 among regions, and the number reported for LVTR is even lower than that of the others.

The level of kidney function in patients starting dialysis varies from country to country and can significantly influence incidence levels ${ }^{7,13}$. However, although it may have influence on the levels registered in Portugal, it is not credible that, unless partially, it could be responsible for such a difference among regions. Despite that, it is something that should be investigated since a higher incidence and better survival, as reported by de Almeida et al, are expected in patients with higher estimated glomerular filtration rate $(\mathrm{GFR})^{5}$.

Among other conditions that are known to be able to influence, in a significant way, the incidence in dialysis, is the level of accessibility to dialysis centers. In Portugal, as already mencioned, there is a network that covers practically the entire territory, especially made up of private satellite hemodialysis centers belonging to major international companies. Portugal has more hemodialysis centers per million inhabitants and per 10,000 km2 of area than most other European countries.

It is in the LVTR that the highest concentration of hemodialysis centers pmp and per $10,000 \mathrm{Km} 2$ - public and private - is found, a concentration much higher than that observed in all other regions. Interestingly, we have found that there is a strong and positive correlation between the number of regional public hemodialysis centers pmp and the regional incidence in dialysis pmp. This means that the greater the number of hemodialysis public centers pmp, the greater the incidence. The number of private hemodialysis centers pmp does not seem to have the same degree of influence, at least in a direct way, which is understandable by the fact that only public hospital centers officially have the possibility of making a decision for patients to start chronic treatment at the hospital itself or at private satellite centers.

The higher incidence in the LVTR may be the result of this higher concentration of hemodialysis centers pmp and per unit area, which is known to increase accessibility and the rates of use of renal replacement therapy. This greater accessibility and availability can change the inclusion criteria in dialysis and usually leads to a greater entry into dialysis for older and more fragile patients and with a greater number of comorbidities. This does not seem to be the situation since, according to data from the PSN Registry, the average age of incident patients has been lower in the LVTR than in other regions.

In the Alentejo Region, where, despite the Alentejo being one of the largest regions in the country, there is only one public hemodialysis center (1.4 pmp versus $3.6 \mathrm{pmp}$ in LVTR), the hemodialysis incidence is the lowest in the country.

Thus, the difference in incidence among regions may lie in the fact that a larger number of public centers allows greater scrutinity in the population regarding CKD and its progressive and more effective 
monitoring. This situation could reduce mortality among CKD patients, which is known to be very high in our country ${ }^{14}$, and allow a greater number to benefit from an effective and appropriate treatment.

\section{CONCLUSION}

In Portugal, the incidence of dialysis in one of the highest in the world and, according to the study carried out by a group of experts appointed by the National Dialysis Monitoring Commission, there is also a remarkable regional difference in incidence. Lisbon and the Tagus Valley Region has extremely high levels of incidence in dialysis, two to three times higher than those registered in the other regions, although in three of them - North, Center and Algarve - the levels are higher than those observed in European countries. Alentejo, the poorest and least populated region, has the lowest incidence in dialysis.

In the analysis we performed, we found that this striking difference among the regions is due exclusively to the fact that there is a much greater entry of patients in hemodialysis. The incidence in peritoneal dialysis is very similar in all regions.

Over the past few years, efforts have been made to find the cause, or causes, responsible for the enormous difference observed in the incidence of dialysis between Portugal and other European countries, causes that are not completely understood. Finding the reason for such large internal difference among regions will certainly be more difficult.

In fact, the usual factors that generate CKD and its faster progression - advanced age, diabetes, hight blood pressure, obesity, excessive salt intake and smoking - do not show different levels of prevalence from region to region. The same is observed in relation to the prevalence of CKD stages 1-5.

Data on the renal function levels of incident patients when entering dialysis are not published. Although it is known that a different GFR criterion should determine the entry into dialysis and can significantly influence the levels of incidence in dialysis, it is not credible that, by itself, it can be fully responsible either for the high incidence of dialysis recorded in the country or for the great difference observed among regions.

Something interesting we found was a close correlation between the number of public dialysis centers (pmp) and the levels of incidence in dialysis (pmp), a correlation that doesn't seem to exist in relation to the number of private centers.

The greater the number of public centers, the greater the incidence. In fact, it is in the LVTR where there is the highest concentration of public centers - $3.6 \mathrm{pmp}$ - that the highest incidence occurs (387 pmp). In Alentejo, where there is only one public center -1.4 pmp the incidence is the lowest (123 pmp).

Could this larger and denser network of dialysis public centers be responsible for the extremely higher levels of incidence in dialysis in LVTR and for what is described as the striking regional difference in dialysis incidence? A definitive answer will only be possible if a wider access to the data contained in the Ministry of Health's Integrated Chronic Kidney Disease Management Platform (PI-GID) is allowed. As this does not currently exist, it is not possible to eliminate the hypothesis that the platform, whose data is not audited, contain errors and omissions, or the possibility of a misinterpretation of the data contained therein.

We must keep in mind that keeping the data confidential, or restricted to a few observers, does not help scientific work, does not allow in clarifying problems or improving clinical results and does not improve health literacy.

\section{Disclosure of potential conflicts of interest: none declared.}

\section{References}

1. Martins Prata M "Incidence in dialysis in Portugal and Spain"

Edition of the author (request by email mateus.mprata2@gmail.com)

2. Stel VS, Brück K, Frazer S, et al. International differences in chronic kidney disease prevalence: a key public health and epidemiological research issue. Nephrol Dial Transplantation 2017;32(supp|2);ii129-ii135

3. Brück K, Stel VS, Gambaro J, et al. CKD prevalence varies across European general population J Am Soc Nephrol 2016;27(7): 2135-2147

4. Martín-Cleary C, Ortiz A. CKD hotspots around the world: where, why and what the lessons. A CKD review series. Clin Kidney J 2014;7:519-523

5. de Almeida E, Raimundo M, Coelho A, Sá H. Incidence, prevalence and crude survival of patients starting dialysis in Portugal (2010-2016): analysis of the National Health System individual Registry. Clin Kidney J 2020;14(3):869-875

6. 2016 PSN Registry of Dialysis and Transplantation.

7. Couchoud C, Guihenneuc C, Bayer F, Stengel B. The timing of dialysis initiation affects the incidence of renal replacement therapy Nephrol Dial Transplant 2010;25:1576-1578

8. Stel VS, Tomsom C, Ansell D et al. Level of renal function in patients starting dialysis: an ERA-EDTA study. Nephrol Dial Transplant 2010; 25:3315-3325

9. Caskey F, Kramer A, Elliott Rf et al. Global variation in renal replacement therapy for end-stage renal disease. Nephrol Dial Transplant 2011;26;2601-2610

10. Portuguese Competition Authority - Analysis of competition conditions in the provision of services of hemodialysis care in Portugal. 2020 Report

11. Stel VS, de Jong RW, Kramer A, Jager KJ, et al. Supplemented ERA-EDTA Registry data evaluated the frequency of dialysis, kidney transplantation, and comprehensive conservative management for patients with kidney failure in Europe doi:10.1016/j.kint.2020.12.010

12. Hörl WH, de Alvaro F, Williams F. Healthcare systems and end-stage renal disease (ESRD) therapies - an international review: access to ESRD treatments. Nephrol Dial Transplant 1999;14(suppl 6):10-15

13. Gonzalez-Espinoza L, Ortiz A. 2012 ERA-EDTA Registry Annual Report Cautious optimism on outcomes, concern about persistent inequalities and data black-out. Clin Kidney J 2015;8(3):243-247

14. Santos J, Fonseca I, Malheiro J, et al. End-stage renal disease versus death in a Portuguese cohort of elderly patients: an approach using competing event analysis. J Invest Med 2017;65:1041-1048

\section{Correspondence to:}

M Martins Prata

E-mail: mateus.mprata2@gmail.com 